\title{
Appliances used in migraine prevention therapy
}

Fitting acrylic occlusal splints and an experimental laminated appliance used in migraine prevention therapy

M. F. Barnes, J. L. Geary, T. J. Clifford and P-J. Lamey Br Dent J 2006; 200: 283-286

\section{Objective}

To evaluate clinical procedures and chair time required to seat and adjust hard, heat-cured acrylic occlusal splints and an alternative laminated appliance developed to simplify construction of migraine prevention appliances.

\section{Design and setting}

Single-centre study in the Oral Medicine Clinic, The Royal Hospitals, Belfast, Northern Ireland.

\section{Method}

Questionnaires were distributed, January-May 2003, to operators fitting occlusal splints for 100 consecutive patients selected for migraine prevention therapy. Half the appliances were made in heat-polymerised acrylic with the remainder using a novel combination of ethylene vinyl acetate and light-curing urethane dimethacrylate. Information on operator experience, the nature of any fitting surface and occlusal adjustments together with an estimate of the time taken to make alterations was recorded.

\section{Key findings}

The need for adjustment to seat appliances intraorally was significantly less for migraine prevention appliances made using an experimental laminating technique. Where modifications were necessary, there was no significant difference in the chair time required to fit either the heatcured hard or experimental laminated migraine prevention appliance. Conclusion

Provision of migraine prevention appliances may be more time efficient if the dental practitioner considers a laminated approach to construction.

\section{IN BRIEF}

- In a busy clinical environment laminated appliances required less chair-side adjustment than acrylic appliances.

- With both appliances we still advise an inter-premolar separation of about $4 \mathrm{~mm}$ but this may be increased if the patient also has marked tooth wear anteriority.

- The principle of the mechanism of action of both appliances is to reduce nocturnal parafunctional activity.

\section{COMMENT}

Hard occlusal stabilisation splints can be difficult to fit. This paper offers a possible solution to this problem using splints with a hard occlusal surface but a resilient fitting surface. When using conventional heat cured acrylic, the hardness of the material, the hardness of enamel, and the large surface area where the two come into contact mean that the slightest chip to the casts, air bubble in the stone or distortion of the impression will result in real problems fitting the final appliance. If you cannot fully seat the splint, the occlusion will be completely wrong too. This is time and money.

The paper describes a small scale and very simple piece of clinical research. Dentists at different grades, who were fitting both hard and laminated appliances, were asked about the need and the time taken for adjustment. The adjusting dentists were understandably not able to be blinded, so there is a significant risk of a biased response which we should be cautious about. Unfortunately neither were the splints truly randomly assigned (for example by random number generation) into the hard or laminated groups, but an alternate assignment was used so hopefully the risk of bias from this source should be minimal. The timings for adjustment were estimated into bands - so there is no objective measure of time to fit which may also be a weakness. Analysis based on actual timings may have been more informative. In the final analysis though, and assuming no operator bias, it does seem that the laminated splints are quicker and easier to fit, with a knock on benefit for occlusal adjustment, and as someone who fits such appliances, that is good to know. Of course, this tells us nothing about the longevity of the final appliance, how they fail or whether there is any difference in response for migraineurs or anyone else; this would need much more work.

Improvements in technology and materials only go some way to making our practising lives easier. If you want a well fitting splint you will still need an excellent and undistorted impression, properly managed and poured, and casts that are handled with real care, irrespective of the material or technique used. A resilient fitting surface might help though, and the technique is well described in the paper so should allow dentists and technicians to decide for themselves.

J. Steele, Consultant in Restorative Dentistry, Newcastle-upon-Tyne Dental Hospital doi: 10.1038/sj.bdj.4813312 